\title{
Relação entre aquíferos susceptíveis à contaminação e Áreas de Preservação Permanente Hídrica em ljuí, RS
}

\author{
Cristiano Niederauer da ROSA ${ }^{1}$, Rutinéia TASSI ${ }^{2}$, Willian Fernando DE BORBA ${ }^{3}$, \\ Jean Ricardo FAVARETTO ${ }^{4}$, Gabriel D'Avila FERNANDES 5 \& José Luiz SILVÉRIO DA SILVA ${ }^{6}$ \\ ${ }^{1}$ Programa de Pós-graduação em Sensoriamento Remoto, Universidade Federal do Rio Grande \\ do Sul. Av. Bento Gonçalves, 9.500, Campus do Vale, CEP 91.501-970, Porto Alegre, RS, Brasil \\ (cristianonrd@gmail.com). \\ 2 Departamento de Engenharia Sanitária e Ambiental, Universidade Federal de Santa Maria. Av. Roraima, \\ 1000, Cidade Universitária, Bairro Camobi, CEP 97.105-900, Santa Maria, RS, Brasil (rutineia@gmail.com). \\ ${ }^{3}$ Departamento de Engenharia e Tecnologia Ambiental, Universidade Federal de Santa Maria. BR 386/ \\ 158, KM 40, Linha 7 de setembro, S/ N, Interior, CEP. 98400-000, Frederico Westphalen, RS, Brasil \\ (borbawf@gmail.com) \\ ${ }^{4}$ Departamento de Engenharias e Ciência da Computação, Universidade Regional Integrada do Alto \\ Uruguai e das Missões, Av. Assis Brasil, 709, Bairro Itapagé, CEP 98400-000, Frederico Westphalen, RS, \\ Brasil (jeanfavaretto@gmail.com). \\ ${ }^{5}$ Programa de Pós-graduação em Engenharia Civil, Universidade Federal de Santa Maria. Av. Roraima, \\ 1000, Cidade Universitária, Bairro Camobi, CEP 97.105-900, Santa Maria, RS, Brasil (borbawf@gmail.com, \\ enggabrielfernandes@gmail.com) \\ ${ }^{6}$ Departamento de Geociências, Universidade Federal de Santa Maria. Av. Roraima, 1000, Cidade \\ Universitária, Bairro Camobi, CEP. 97.105-900, Santa Maria, RS, Brasil (silverioufsm@gmail.com).
}

\begin{abstract}
Resumo. A água subterrânea, embora possa estar protegida pelo meio natural, também pode ser contaminada, especialmente em razão de aterros sanitários, despejos industriais e domésticos, atividades agrícolas e minerais, entre outras. Nesse contexto, esse estudo teve por objetivo avaliar a susceptibilidade à contaminação das águas subterrâneas no município de ljuí no oeste do Rio Grande do Sul utilizando-se do método IS (índice de susceptibilidade) frente às áreas de preservação permanente hídricas (APPh). A susceptibilidade foi determinada a partir do método IS, que avalia os parâmetros de recarga, profundidade do nível freático, declividade, material do aquífero e uso do solo. Devido à importância das APPh na recarga do aquífero, estas foram identificadas e analisadas com especial interesse. O índice de susceptibilidade indicou que 0,36\% da área total do município apresentou susceptibilidade classificada como muito baixa, 11,22\% como baixa, 50,12\% de moderada à baixa, 37,97\% de moderada à alta e apenas 0,30\% elevada. Cerca de $7 \%$ da área total do município foi identificada como área de preservação permanente, sendo que deste percentual, a maior parcela encontra-se na classe de susceptibilidade de moderada à alta (3,29\%), e o restante das áreas de preservação permanente se enquadraram na classe de susceptibilidade baixa. A ferramenta metodológica utilizada mostrou-se importante na identificação de áreas de maior susceptibilidade à contaminação do aquífero, e a relevância da manutenção das áreas de preservação permanente no município de ljuí, RS.
\end{abstract}

Palavras-chave. água subterrânea, aquífero, SASG.

\begin{abstract}
AQUIFER SUSCEPTIBILITY TO CONTAMINATION AND ITS RELATIONSHIP WITH WATER PERMANENT PRESERVATION AREAS, IN IJUÍ, RS. Groundwater, although protected by the natural environment, can also be contaminated, especially due to landfills, industrial and domestic waste, agricultural and mineral activities, among others. In this context, this study aimed to evaluate the susceptibility to groundwater contamination in the municipality of ljuí, Western Rio Grande do Sul, by using the SI (susceptibility index) method in order to evaluate hydric permanent preservation areas (hPPA). The SI was determined evaluating recharge parameters, groundwater depth, slope, aquifer material and land use. Due to the importance aquifer recharge for hPPA the main analysis was focused on this special issue. The susceptibility index indicated that $0.36 \%$ of the total area of the municipality was classified as very low susceptibility, $11.22 \%$ as low susceptibility, $50.12 \%$ as moderate to low susceptibility, $37.97 \%$ as moderate to high susceptibility and only $0,30 \%$ high susceptibility. About $7 \%$ of the total area of the municipality was identified as PPA, and the largest proportion (3.29\%) is in the moderate to high susceptibility class, while the remaining portion in the low susceptibility class. The methodological procedure used allowed to identify areas of greater susceptibility to aquifer contamination, and the importance of maintaining permanent preservation areas in the municipality of ljuí, RS in order to keep the groundwater quantity and quality.
\end{abstract}

Keywords. Groundwater, aquifer, SASG. 


\section{Introdução}

Toda a água que ocorre abaixo da superfície da Terra, preenchendo os poros ou vazios das rochas sedimentares, fraturas, falhas e fissuras das rochas vulcânicas é denominada subterrânea, e sua produtividade e/ou a proteção natural está estritamente relacionado com o material de formação do meio aquífero (ABAS, 2019). Trata-se de um recurso de alta qualidade natural, que exige pouco tratamento sanitário e tem baixo custo de captação (CETESB, 2014).

Como consequência, a água subterrânea vem se tornando essencial para diversos usos, destacando sua importância. Entretanto, a facilidade com a qual ocorre a exploração dessas águas, por meio da perfuração de poços e subtração da água com bombas submersas, causa preocupação para o gerenciamento dos recursos hídricos (Custódio \& Silva Júnior, 2008). Soma-seaisso, o descarte da carga contaminante, gerada por atividades antrópicas como urbanização, indústria, agricultura e mineração, que é inadequadamente controlada, e possui certos componentes que excedem a capacidade de atenuação das camadas do solo, prejudicando as águas subterrâneas (Foster et al., 2002). A contaminação das águas subterrâneas tem como consequências problemas relacionados ao abastecimento público, principalmente em locais em que não há a rede pública de abastecimento como comunidades rurais e pequenos núcleos populacionais.

Desta forma, é importante que se tenha o conhecimento mais detalhado das atividades humanas com potencial de poluição das águas subterrâneas, como os vazamentos de esgotos, tanques de combustível, lançamento de efluentes industriais em superfície, lagoas de efluentes de indústrias, áreas de cultivo agrícola em que se tem o uso de agrotóxicos e criação de animais (Foster \& Hirata, 1991).

Com o intuito de proteger as águas subterrâneas, que cada vez se consolidam mais como uma alternativa estratégica para suprir a atual demanda de abastecimento humano (Shrestha et al., 2016), o Estado do Rio Grande do Sul, por meio do Decreto Estadual No 42.047/2002 (Estado do Rio Grande do Sul, 2002), alterado pelo
Decreto Estadual No 52.035/2014 (Estado do Rio Grande do Sul, 2014), relativo ao gerenciamento das águas subterrâneas e dos aquíferos em seu artigo 26, salienta que: "Todos os projetos de implantação ou ampliação de empreendimentos que apresentem riscos de poluição das águas subterrâneas deverão conter estudos detalhados de caracterização hidrogeológica e de vulnerabilidade dos sistemas aquíferos, bem como projeto de medidas de proteção, controle e monitoramento a serem adotados e aprovados pela Fundação Estadual de Proteção Ambiental Henrique Luiz Roessler (FEPAM)".

A vulnerabilidade dos sistemas aquíferos representa a facilidade ou dificuldade de um contaminante advindo de atividades antrópicas (pontuais ou difusas) migrar da superfície do terreno até a zona saturada do aquífero (Menezes et al., 2013; Muhammad et al., 2015). Assim, com o intuito de aprimorar a gestão e conservação deste recurso natural (Menezes et al., 2013; Coelho et al., 2013), o mapeamento para identificação da fragilidade do meio aquífero à contaminação é um produto essencial, no qual as ferramentas de geoprocessamento podem ser utilizadas para indicar locais que necessitam uma maior proteção de maneira eficaz (Löbler et al., 2013).

Contudo, a qualidade das águas subterrâneas depende do meio ambiente como um todo, o que torna a preservação de toda a área de influência uma peça chave para a manutenção dos recursos renováveis. Com esse intuito, surgiu a Lei Federal No 12.651/2012, criada para dar proteção a áreas, cobertas ou não por vegetação nativa, com a função ambiental de preservar os recursos hídricos, a paisagem, a estabilidade geológica e a biodiversidade, facilitando o fluxo gênico de fauna e flora, proteger o solo e assegurar o bem-estar das populações humanas (Brasil, 2012).

Considerando o aumento das atividades potencialmente degradantes do meio ambiente e das águas subterrâneas, principalmente devido a atividades antrópicas realizadas na superfície da terra, o presente estudo teve como objetivo avaliar a susceptibilidade das águas subterrâneas à contaminação. A metodologia do índice de susceptibilidade (IS) (Ribeiro, 2005) foi aplicada 
ao município de ljuí-RS, permitindo verificar especialmente a vulnerabilidade dos aquíferos associada às áreas de preservação permanente.

\section{Local de estudo, materiais e métodos}

\subsection{Local de estudo}

O município de ljuí está localizado na região Noroeste do Estado do Rio Grande do Sul, e possui uma população de aproximadamente 79 mil habitantes, ocupando uma área de 689.387 $\mathrm{km}^{2}$ (IBGE, 2010). Segundo informações da SEMA (2004), essa área está situada na Região Hidrográfica do Uruguai $(U)$, Bacia Hidrográfica do Rio ljuí U-90, como pode observado na Figura 1, com impacto direto nas condições da bacia hidrográfica (Scheeren, 2008).

O clima da região é do tipo subtropical II, medianamente úmido, com variação longitudinal das temperaturas médias (Rossato, 2011). As atividades econômicas desenvolvidas na Bacia Hidrográfica do Rio ljuí U-90, estão ligadas ao setor primário, predominando a agricultura, com destaque para as lavouras de soja e milho
(FEPAM, 2016).

O município está inserido na Bacia Sedimentar do Paraná, Formação Serra Geral (FSG), Fácies Gramado (K1_beta_gr) e Paranapanema (K1_beta_pr), sendo que a FSG é proveniente de derrames basálticos (rochas vulcânicas) (CPRM, 2006). Considerando aspectos hidrogeológicos, na área de estudo tem-se o Sistema Aquífero Serra Geral (SASG I), e a hidroestratigrafia constitui principalmente de litologias basálticas, amigdaloides e fraturadas, capeadas por espesso solo avermelhado. Assim, os solos presentes na região são originários de rochas basálticas e seus produtos de intemperização (Machado \& Freitas, 2005).

\subsection{Materiais e métodos}

\subsubsection{Determinação do Índicede Susceptibilidade (IS)}

Perante a quantidade de informações consideradas no presente estudo, foi necessária a criação de um banco de informações geográfıcas, que possibilitasse o acesso, edição e

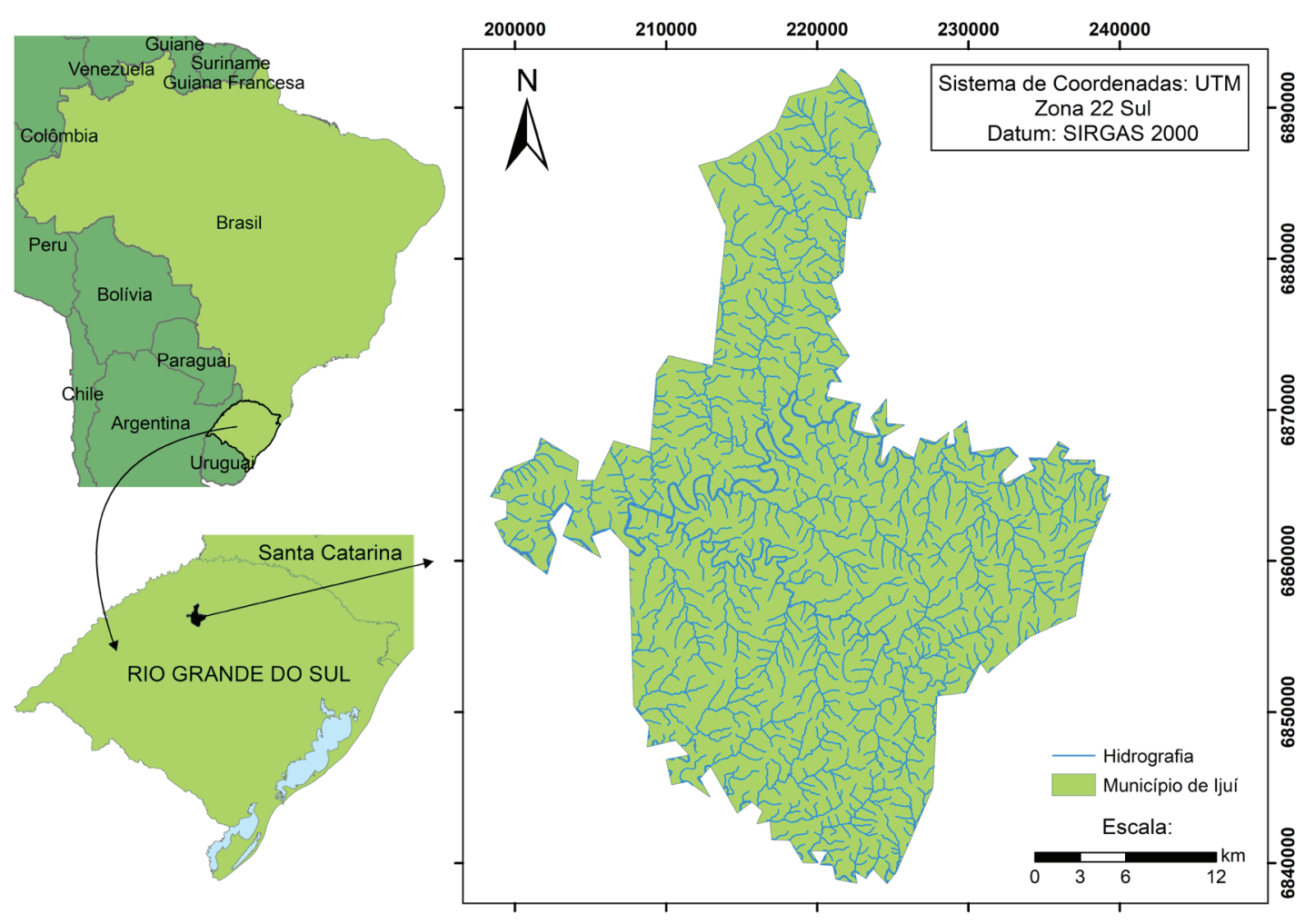

Figura 1. Localização do Município de ljuí, RS.

Figure 1. Location of the Municipality of Ijuí, RS. 
manipulação destes dados. Sendo assim, nesse processo foi fundamental a utilização de um SIG (Sistema de Informação Geográfica).

O método IS, desenvolvido por Ribeiro (2005), é uma adaptação do método DRASTIC desenvolvido por Aller et al. (1987). Nessa adaptação foi considerado, além dos parâmetros recarga $(R)$, profundidade do nível freático (D), declividade $(T)$, material do aquífero $(A)$, o parâmetro uso do solo (LU). Desse modo, a determinação do IS (Ribeiro, 2005) é realizada a partir dos parâmetros apresentados na tabela 1 e equação 1, a qual foi inserida no SIG na ferramenta Raster calculator. Os demais procedimentos para a obtenção e classificação das variáveis que compõem o método IS são descritos detalhadamente na sequência.

Tabela 1. Parâmetros recarga $(R)$, profundidade do nível freático $(D)$, declividade $(T)$, material do aquífero $(A)$ e uso do solo (LU) com seus respectivos fatores de ponderação de acordo com Ribeiro (2005) para adeterminação do IS. Table 1. Parameters recharge (R), groundwater depth (D), slope $(T)$, aquifer material $(A)$ and land use $(L U)$ with their respective and weighting factors according to Ribeiro (2005) for a171 IS determination.

\begin{tabular}{lccccc}
\hline Parâmetro & D & R & A & T & LU \\
\hline Fator de ponderação & 0,186 & 0,212 & 0,259 & 0,121 & 0,222 \\
\hline
\end{tabular}

$$
\text { IS }=0,186 D+0,212 R+0,256 A+0,121 T+0,222 L U
$$

Onde: D - Profundidade do nível freático; $\mathrm{R}$ - Recarga; A - material do aquífero; T declividade e LU - uso do solo.

Na estimativa do parâmetro $R$ (recarga) do SASG I, foram utilizados os valores propostos por Hausmann (1995) de 175 mm anuais. Considerando-se que a precipitação média anual na região é de 1.445,00 mm (Bergamaschi et al., 2004), tem-se um valor de $12,10 \%$ de recarga oriunda da precipitação. Esse parâmetro é importante, visto que considera a quantidade de água que irá chegar até o aquífero, ou seja, quanto maior a recarga, maior será sua susceptibilidade.

Para a determinação de A e D foram utilizadas as informações disponíveis no banco de dados do Sistema de Informações de Águas Subterrâneas (SIAGAS), mantido pela Companhia de Pesquisa de Recursos Minerais (CPRM), disponível no endereço: http://siagasweb. cprm.gov.br/layout/ (SIAGAS, 2016). Assim, utilizou-se as informações oriundas da data de perfuração de 235 poços tubulares, localizados nos munícipios gaúchos de Ajuricaba, Augusto Pestana, Catuípe, Chiapeta, Coronel Barros, Cruz Alta, Ijuí e Pejuçara. Foram utilizadas as informações destes municípios da região, devido à quantidade limitada de poços localizados no município de ljuí, que não representaram de forma satisfatória as condições hidrogeológicas da região.

Os parâmetros $\mathrm{D}$ e $\mathrm{T}$, representam uma menor interferência no método, pois são dependentes de outras variáveis, como por exemplo o material do aquífero e a recarga. Porém, quanto mais próximo da superfície o nível da água subterrânea, mais susceptível à contaminação. 0 mesmo ocorre para a topografia, quanto menor a declividade da superfície do terreno, maior será a sua susceptibilidade. Isso ocorre devido ao maior acúmulo de líquidos na superfície ocasionado por essa condição, e um maior tempo para percolar da zona aerada até a zona vadosa. Já o parâmetro LU está relacionado com a proteção do meio às atividades realizadas. Por exemplo, o uso do solo por área urbana apresenta maior risco de contaminação quando comparada à área natural.

Deste modo, as informações de R, D e A obtidas de maneira pontual, conforme supracitado, foram interpoladas para a estimativa de seus valores em locais não amostrados. Neste processo, aplicou-se um método determinístico e local, denominado IDW (Inverse Distance Weighting), no qual os valores interpolados nunca atingem o valor máximo ou mínimo das amostras, o que o torna também um interpolador exato (Johnston et al., 2003). Apesar de possuir algumas limitações, este método é um dos mais utilizados pelos pesquisadores e estudiosos da área de hidrogeologia, sendo encontrada sua aplicação em diversos trabalhos (Tavares et al., 2009; Arcos et al., 2014; Terra et al., 2016a, 2016b; Löbler et al., 2016; Borba et al., 2016).

O parâmetro LU foi obtido a partir de uma imagem multiespectral datada de junho de 2015, sem a presença de nuvens, pertencente a missão Landsat 8, com resolução espacial de 30,00 m (NASA, 2013), disponível em: http://earthexplorer. 
usgs.gov/. Essa imagem foi submetida a um processo de classificação automática de imagens por meio da ferramenta Raster Classification, na qual foram consideradas as classes: área urbana; água; florestas e zonas semi-naturais; e pastagens e áreas agro-florestais.

As declividades, parâmetro $T$, foram geradas a partir das curvas de níveis obtidas através da base cartográfica vetorial contínua do Estado do Rio Grande do Sul (Hasenack \& Weber, 2010), processadas por meio de modelagem com a ferramenta Topo to Raster. Essa modelagem permite a criação de modelos considerados hidrologicamente corretos, por respeitar restrições que garantam uma estrutura de drenagem conectada e mais precisa (Marcuzzo et al., 2011).
As informações de D, R, A, LU e T, foram reclassificadas de acordo com as pontuações apresentadas nas tabelas 2 e 3, por meio da ferramenta Reclassify. Os valores de D, R, A e $\mathrm{T}$, foram ampliados em 10 vezes, conforme sugerido por Veríssimo (2010). Esse autor indica que esse procedimento permite uma melhor visualização das variáveis, quando comparadas ao parâmetro LU.

A classificação final dos valores de IS (Ribeiro, 2005) foi proposta por Stigter et al. (2006), conforme apresentado na tabela 4. Podese perceber que a classificação dos valores varia entre menores que $30,00 \%$ a maiores que $90,00 \%$, sendo que cada classe representa uma condição de susceptibilidade à contaminação.

Tabela 2. Parâmetros D, R e T do índice DRASTIC (Aller et al., 1987) e suas respectivas pontuações. Table 2. Parameters $D, R$ and T of the DRASTIC index (Aller et al., 1987) and their respective scores.

\begin{tabular}{|c|c|c|c|c|c|}
\hline \multicolumn{6}{|c|}{ Parâmetros } \\
\hline D & & $\mathbf{R}$ & & $\mathbf{T}$ & \\
\hline Profundidade (m) & Pontuação & Recarga (mm/ano) & Pontuação & Declividade (\%) & Pontuação \\
\hline$<1,5$ & 10 & $<51$ & 1 & $<2$ & 10 \\
\hline $1,5-4,6$ & 9 & $21-102$ & 3 & $2-6$ & 9 \\
\hline $4,6-9,1$ & 7 & $102-178$ & 6 & $6-12$ & 5 \\
\hline $9,1-15,2$ & 5 & $178-254$ & 8 & $12-18$ & 3 \\
\hline $15,2-22,9$ & 3 & \multirow{3}{*}{$>254$} & \multirow{3}{*}{9} & \multirow{3}{*}{$>18$} & \multirow{3}{*}{1} \\
\hline $22,9-30,5$ & 2 & & & & \\
\hline$>30,5$ & 1 & & & & \\
\hline
\end{tabular}

Tabela 3. Parâmetros A e LU e suas respectivas pontuações, conforme Aller et al. (1987) e Ribeiro (2005).

Table 3. Parameters A and LU and their respective scores according to Aller et al. (1987) and Ribeiro (2005).

\begin{tabular}{|c|c|c|c|c|}
\hline \multicolumn{5}{|c|}{ Parâmetros } \\
\hline \multicolumn{3}{|l|}{$\mathbf{A}$} & \multicolumn{2}{|l|}{$\mathbf{L U}$} \\
\hline Natureza do aquífero & Pont. & Usual & Ocupação do solo & Pont. \\
\hline Xisto argiloso, argilito & $1-3$ & 2 & Áreas agrícolas & \\
\hline Rocha metamórfica/ígnea & $2-5$ & 3 & Perímetro de rega (Culturas anuais) & 90 \\
\hline $\begin{array}{c}\text { Rocha metamórfica/ígnea } \\
\text { alterada }\end{array}$ & $3-5$ & 4 & Culturas permanentes (vinhas...) & 70 \\
\hline Till glacial & $4-6$ & 5 & agroflorestais, áreas agrícolas heterogêneas & 50 \\
\hline $\begin{array}{l}\text { Arenito, calcário e } \\
\text { argilito estratificados }\end{array}$ & $5-9$ & 6 & $\underline{\text { Áreas artificiais }}$ & \\
\hline Arenito maciço & $4-9$ & 6 & Descargas de resíduos artificiais e aterros & 100 \\
\hline Calcário maciço & $4-9$ & 8 & ciros, áreas minerais a céu aberto & 80 \\
\hline Areia a basalto & $4-9$ & 8 & Áreas urbanas contínuas, aeoroportos & 75 \\
\hline Basalto & $2-10$ & 9 & Áreas urbanas descontínuas & 70 \\
\hline \multirow{3}{*}{ Calcário carsificado } & \multirow{3}{*}{$9-10$} & \multirow{3}{*}{10} & Áreas naturais & \\
\hline & & & Ambientes aquáticos (sapais, salinas..) & 50 \\
\hline & & & Florestas e zonas semi-naturais & 0 \\
\hline
\end{tabular}


Tabela 4. Classificação final do IS segundo Stigter et al (2006).

Table 4. Final IS classification according to Stigter et al. (2006).

\begin{tabular}{cc}
\hline Valores (\%) & Susceptibilidade \\
\hline$>90$ & Extremamente vulnerável \\
\hline $80-90$ & Muito elevada \\
\hline $70-80$ & Elevada \\
\hline $60-70$ & Moderada a alta \\
\hline $50-60$ & Moderada a baixa \\
\hline $40-50$ & Baixa \\
\hline $30-40$ & Muito baixa \\
\hline$<30$ & Extremamente baixa \\
\hline
\end{tabular}

\subsubsection{Determinação das áreas de preservação permanente hídricas}

A delimitação das APPh (áreas de preservação permanente hídricas) foi embasada no Código Florestal Brasileiro, Lei nº 12.651/2012 (Brasil, 2012), que apresenta diversos tipos de áreas a serem preservadas. Nesse estudo, foram consideradas apenas as APPh classificadas como curso d'água perene, devido às resoluções dos dados espaciais utilizados.

Todo o processo de delimitação das APPh considerou a base cartográfica vetorial contínua do Estado do Rio Grande do Sul (Hasenack \& Weber, 2010), e seguiu basicamente a metodologia descrita por Peluzio et al. (2010). Como diferencial, neste trabalho a forma de obtenção dos cursos d'água perene foi realizada por meio de uma consulta à simbologia dos dados de hidrografia advindos de Hasenack \& Weber (2010).

\section{Resultados e discussões}

\section{1 Parâmetros $D, R$, A e T}

A figura 2 apresenta a variação espacial dos parâmetros $D, R, A$ e $T$, respectivamente. Em relação ao material do aquífero, tem-se a presença de rochas basálticas da Formação Serra Geral, descritas pela CPRM (2006). Assim, essas rochas possuem basaltos em sua composição, onde segundo OEA/PEA/GEF (2009) essa área está entre a Zona de Confinamento Próximo (ZC), ou seja, até 100 m, e Zona de Forte Confinamento
(ZFC), com espessuras de basalto superiores a $100 \mathrm{~m}$.

As classes de declividade, conforme proposto por Aller et al. (1987) e Ribeiro (2005), estão apresentadas na tabela 5. A partir da análise, pode-se perceber que a área não apresenta, em sua maior parte, topografia irregular, indicando uma área plana. Em áreas planas, as pontuações indicativas de susceptibilidade são maiores, devido ao acúmulo de água superficial, pois, neste caso, o contaminante pode infiltrar no solo e atingir o lençol freático, com maior facilidade.

Tabela 5. Classes de declividade ( $T$ ) presente no município de ljuí, RS.

Table 5. Classes of declivity $(T)$ present in the municipality of ljuí, $R S$.

\begin{tabular}{ccc}
\hline Declividade (\%) & Área (ha) & Área (\%) \\
\hline$<\mathbf{2}$ & $18.335,83$ & 20,25 \\
$\mathbf{2}-\mathbf{6}$ & $28.811,45$ & 31,82 \\
$\mathbf{6}-\mathbf{1 2}$ & $27.860,28$ & 30,77 \\
$\mathbf{1 2} \mathbf{- 1 8}$ & $12.306,02$ & 13,59 \\
$\mathbf{>} \mathbf{1 8}$ & $3.221,93$ & 3,55 \\
\hline
\end{tabular}

\subsection{Parâmetro LU}

A tabela 6 e a figura 3 ilustram as classes de uso do solo presentes no município de ljuíRS. Como pode ser observado, destacam-se como classes predominantes as pastagens e áreas agroflorestais. Segundo FEPAM (2016), na Bacia Hidrográfica do Rio ljuí, a principal atividade econômica da região é a agricultura. A maior parte da população reside na zona rural do município, com propriedades com área média de 20 ha, utilizando a força de trabalho familiar (Lindner, 2007). Essas informações confırmam a coerência do mapa de uso do solo.

Segundo PLAMSAB (2011), há no município a ocorrência de lavouras temporárias com produção de soja e trigo, o que deve servir de alerta para os gestores do município, tendo em vista que, segundo Grabau \& Chen (2016), cultivos deste tipo apresentam diferentes impactos no solo entre si devido a adsorção de nutrientes e pesticidas. Por esse motivo, Foster \& Hirata (1991) alertam que certas práticas agrícolas são capazes de causar contaminação difusa, podendo ocasionar um aumento da salinidade das águas subterrâneas, especialmente em regiões mais áridas. 


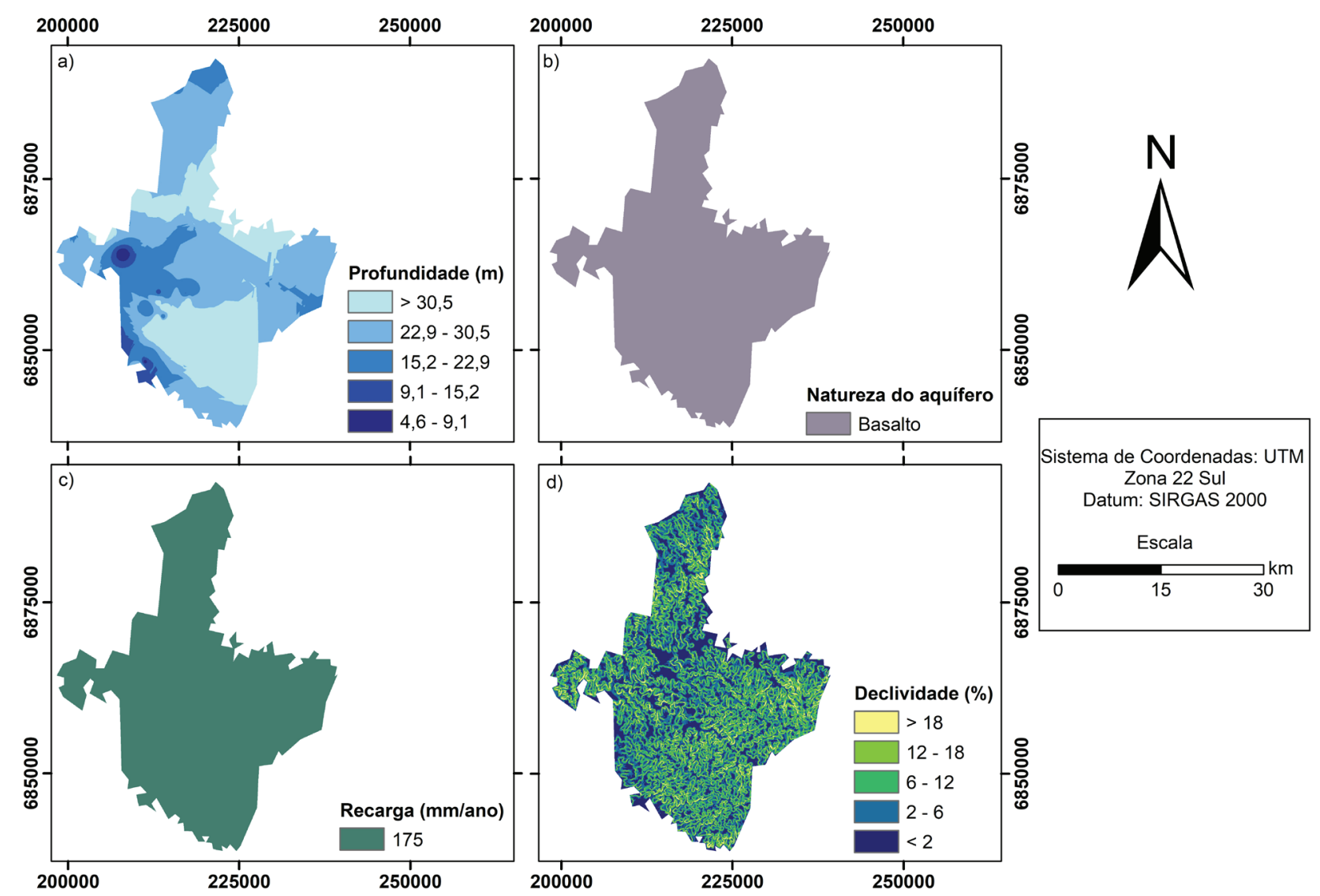

Figura 2. Espacialização dos parâmetros utilizados no cálculo do ISD R, A e T no município de ljuí, RS. A) Parâmetro profundidade do nível freático (D); B) Parâmetro recarga (R); C) Parâmetro material do aquífero (A); D) Parâmetro declividade $(\mathrm{T})$.

Figure 2. Spatialization of the parameters used to calculate the ISD R, A and T in the municipality of ljuí, RS. A) Parameter depth of water table (D); B) Reload parameter (R); C) Aquifer material parameter (A); D) Slope parameter (T).

\subsection{Estimativa do IS}

A figura 4 ilustra as classes de susceptibilidade pelo método IS (Ribeiro, 2005). De acordo com a tabela 7 , pode ser observado um predomínio das classes moderada a baixa (50,15\%) e moderada a alta (37,97\%).

Ribeiro (2005) e Stigter et al. (2006) identificaram o predomínio de valores de susceptibilidade baixa entre 40 e $50 \%$ em áreas com predomínio de uso agrícola, com nível freático próximo a superfície, e formação rochosa com permeabilidade intermediária. Apesar do município de ljuí também apresentar vocação agrícola, algumas características morfométricas e morfológicas da bacia hidrográfica em questão podem estar ocasionando maior vulnerabilidade na área estudada, resultando em IS de moderado baixo a alto em mais de 60\% de sua área.

Em alguns estudos realizados recentemente no Estado do Rio Grande do Sul, com o intuído de avaliar a vulnerabilidade à contaminação de aquíferos pelo método GOD (Löbler et al., 2016; Terra et al., 2016b), foram encontradas vulnerabilidades variando em sua maioria entre baixa e média na bacia hidrográfica do Rio Santa Maria e no Município de São Sepé. Condições menos vulneráveis são encontradas em Canoas e na bacia hidrográfica do rio Passo Fundo (vulnerabilidade baixa em 94,12 \% da área total do município e $73,15 \%$ da área da bacia hidrográfica na classe insignificante) (Feron \& Reginato, 2014; Borba et al., 2016). O contrário Tabela 6. Classes de uso do solo quantificadas no município de ljuí-RS.

Table 6. Quantified classes of land use in the municipality of ljuí, $R S$.

\begin{tabular}{ccc}
\hline Classes & Área (ha) & Área (\%) \\
\hline Florestas e zonas seminaturais & $14.770,61$ & 16,37 \\
\hline Pastagens e áreas agroflorestais & $71.467,83$ & 79,21 \\
\hline Ambientes aquáticos & 618,67 & 0,68 \\
\hline Áreas urbanas & $3.359,79$ & 3,72 \\
\hline
\end{tabular}




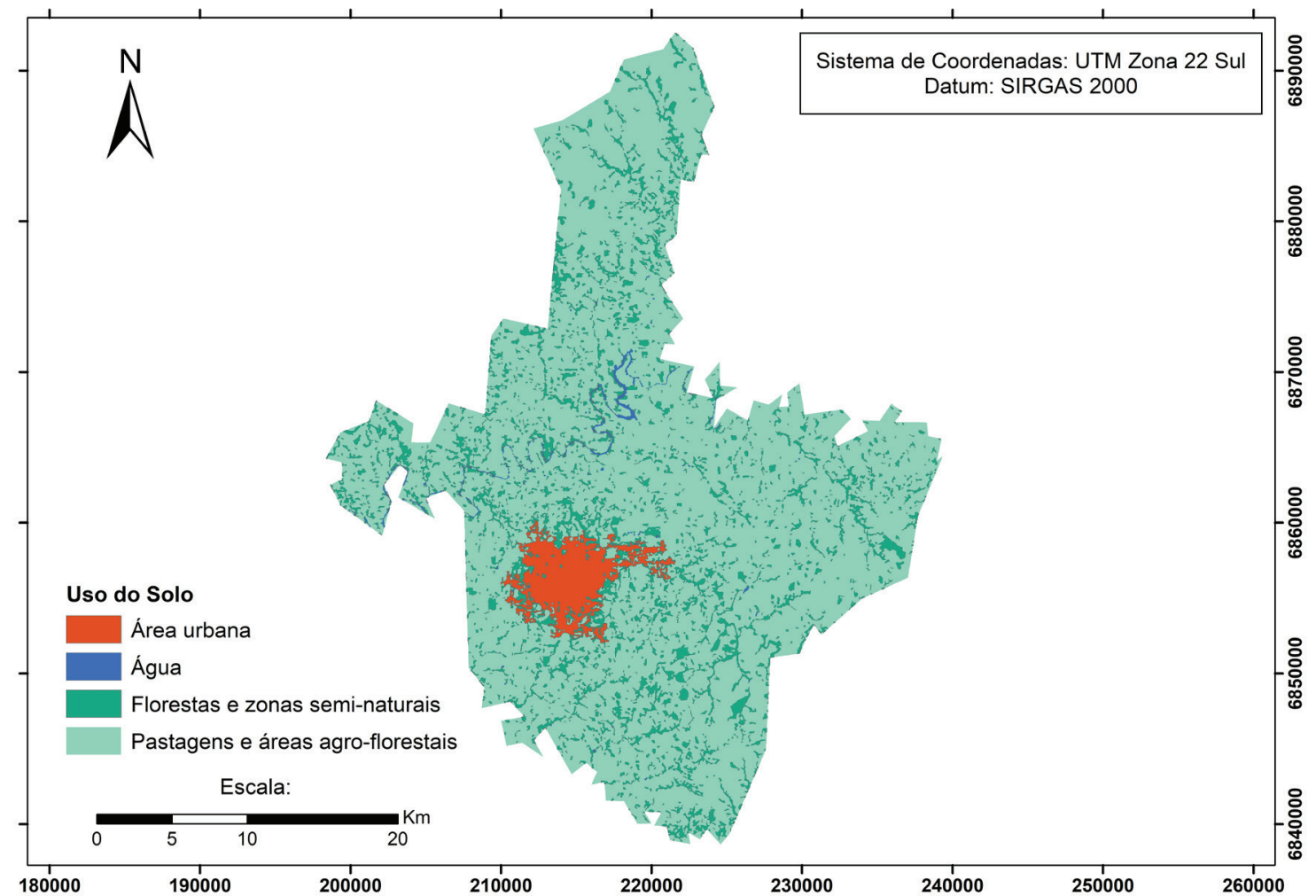

Figura 3. Classes de uso do solo no município de ljuí, RS.

Figure 3. Classes of land use in the municipality of ljuí, RS.

Tabela 7. Áreas referentes às classes de susceptibilidade no município de ljuí, RS.

Table 7. Areas related to the susceptibility classes in the municipality of ljuí, RS.

\begin{tabular}{cccc}
\hline Classes & Valores (\%) & Área (ha) & Área (\%) \\
\hline Muito baixa & $30-40$ & 332,34 & 0,36 \\
Baixa & $40-50$ & $10.128,04$ & 11,22 \\
Moderada a baixa & $50-60$ & $45.226,93$ & 50,12 \\
Moderada a alta & $60-70$ & $34.268,82$ & 37,97 \\
Elevada & $70-80$ & 274,44 & 0,30 \\
\hline
\end{tabular}

ocorre nos municípios de Rosário do Sul - RS e Nova Palma - RS, com vulnerabilidades entre média e alta (Dambrós et al., 2011; Löbler \& Silvério da Silva, 2015).

A partir da estimativa da vulnerabilidade à contaminação do aquífero por meio do IS ao norte do Estado do Rio Grande do Sul-Brasil, no município de Frederico Westphalen, Borba et al. (2016) tiveram como resultado valores classificados como muito baixo a elevado (39\% a 77\%). Simas (2014), em estudo realizado no Sistema Aquífero da Bacia do Tejo - Sado, obteve valores que variaram de extremamente baixo a extremamente vulnerável, com predomínio da classe moderada a baixa (50 - 60\%). Complementarmente, estes autores afirmam que em seu estudo, as áreas com valores entre 30 e $50 \%$ de susceptibilidade, ocorreram devido a uma recarga por precipitação baixa, nível freático muito profundo e uso do solo, sendo realizadas atividades de agricultura tradicional.

\section{4 Áreas de Preservação Permanente Hídricas \\ O município de ljuí teve aproximadamente} $7 \%$ de sua área classificada como APPh (Fig. 5). Cabe salientar que não foi possível classificar as demais APP elencadas por Brasil (2012), em virtude da resolução espectral da base de dados, bem como a indisponibilidade de dados de altimetria com resolução adequadas.

Nas APPh observa-se a presença de áreas naturais (3,35\%), áreas agrícolas (3,52\%) e área urbanizada $(0,13 \%)$, conforme figura 6 . Os resultados encontrados neste estudo alertam sobre a importância do manejo e uso sustentável do solo no local, principalmente se considerado os resultados do IS nas APPh (Fig. 7), e o fato de 3,29\% do total de área de preservação do município estar englobada na classe de susceptibilidade moderada à alta, conforme apresentado na tabela 8. 


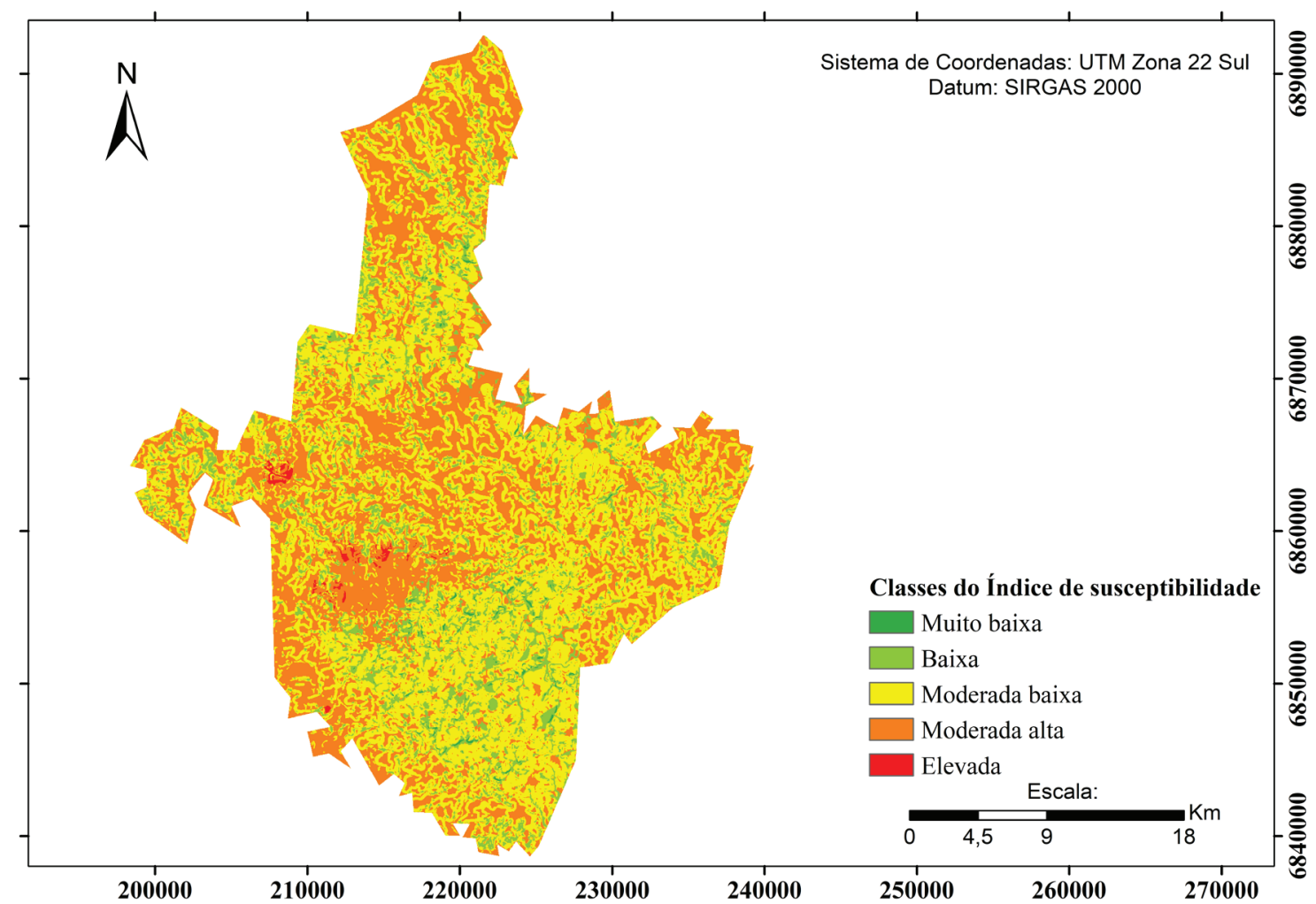

Figura 4. Espacialização da estimativa do IS no município de Ijuí, RS.

Figure 4. Spatialization of IS estimation in the municipality of Ijuí, RS.

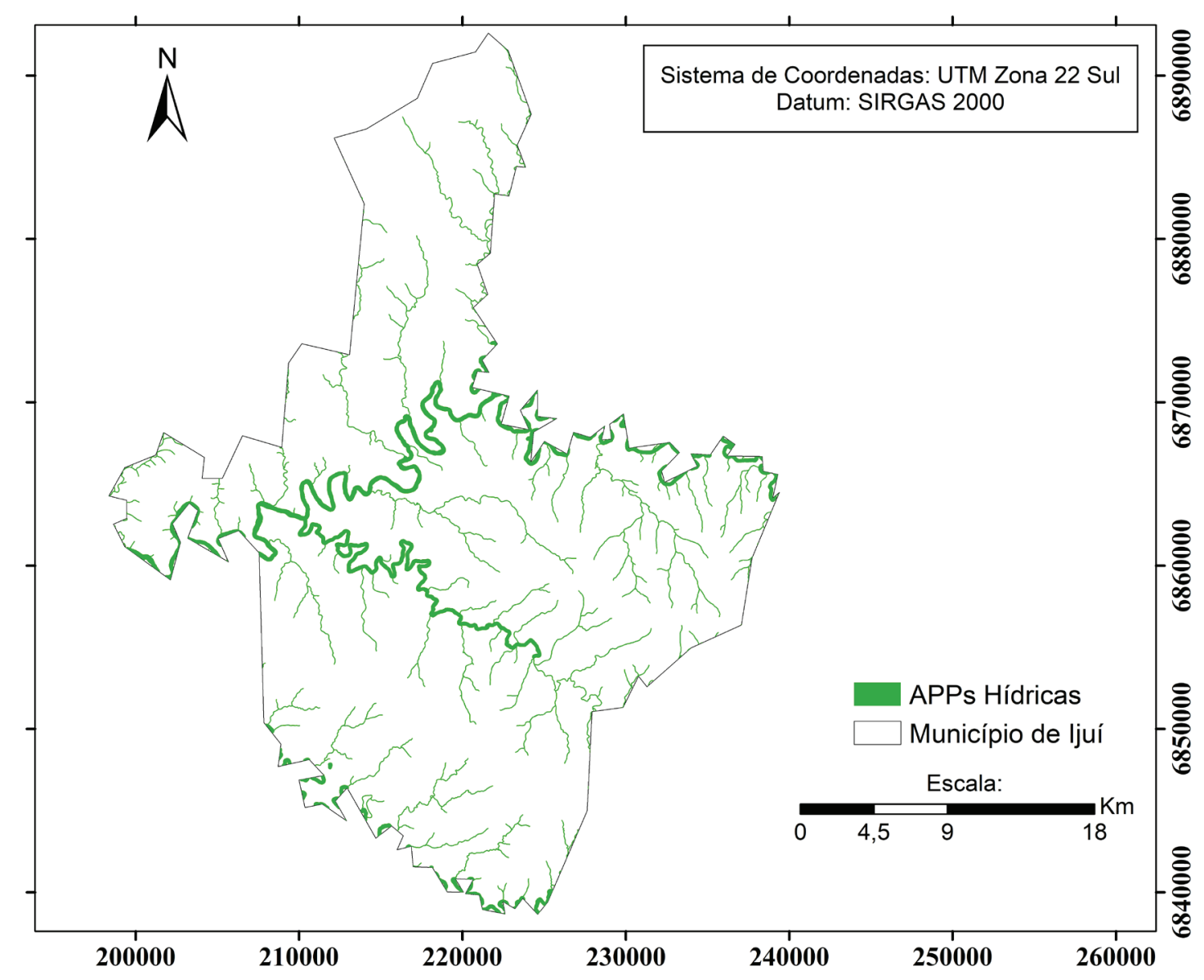

Figura 5. APPh do município de ljuí, RS.

Figure 5. APPh of the municipality of Ijuí, RS. 


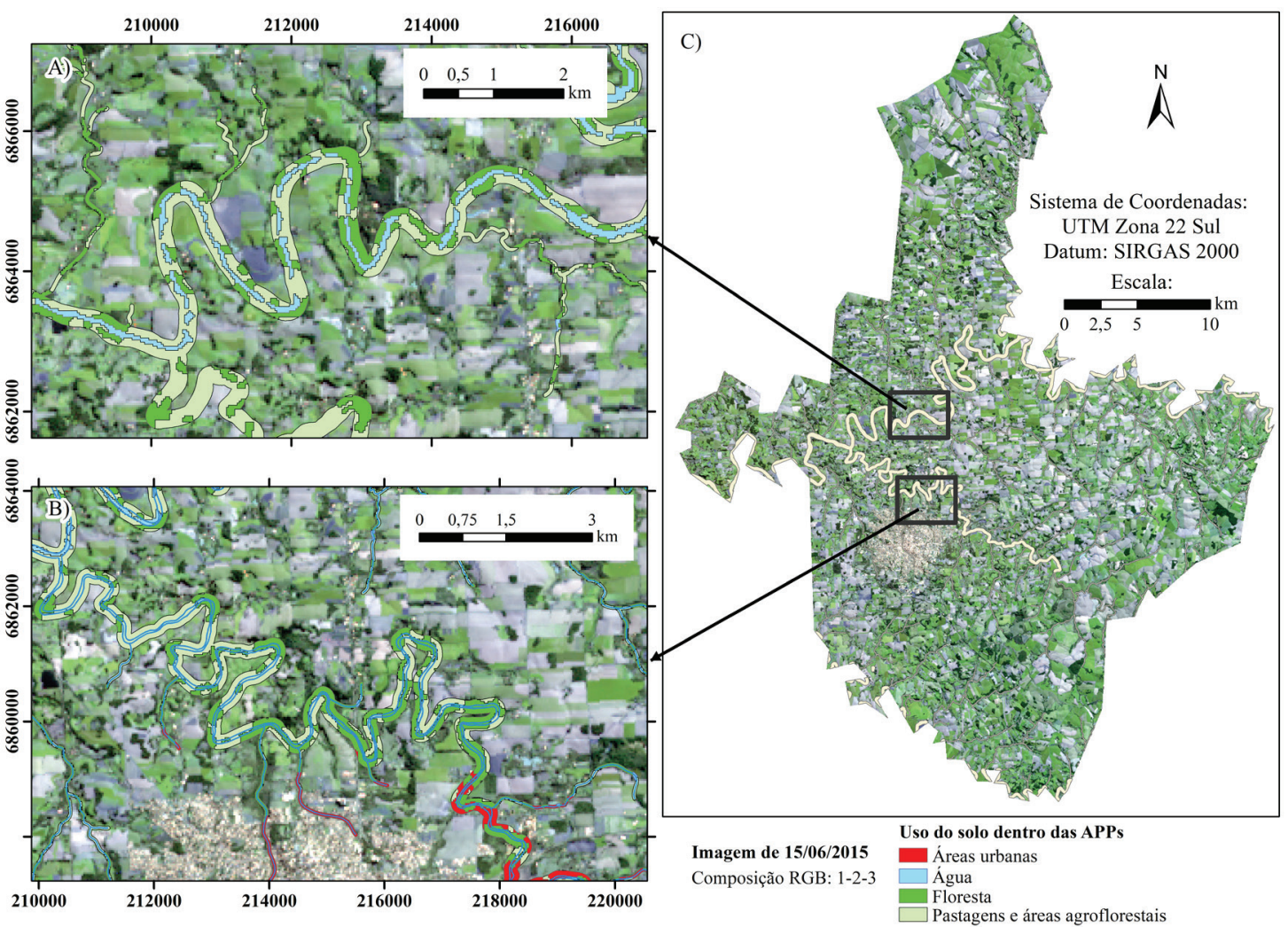

Figura 6. Uso da terra dentro das APPh do município de ljuí, RS. A) Ampliação em área Rural; B) Ampliação em área urbana; C) Panorama geral das APPh em ljuí, RS.

Figure 6. Land use within the APPh of the municipality of Ijuí, -RS. A) Extension in rural area; B) Extension in urban area; C) Overview of the APPh in Ijuí, RS.
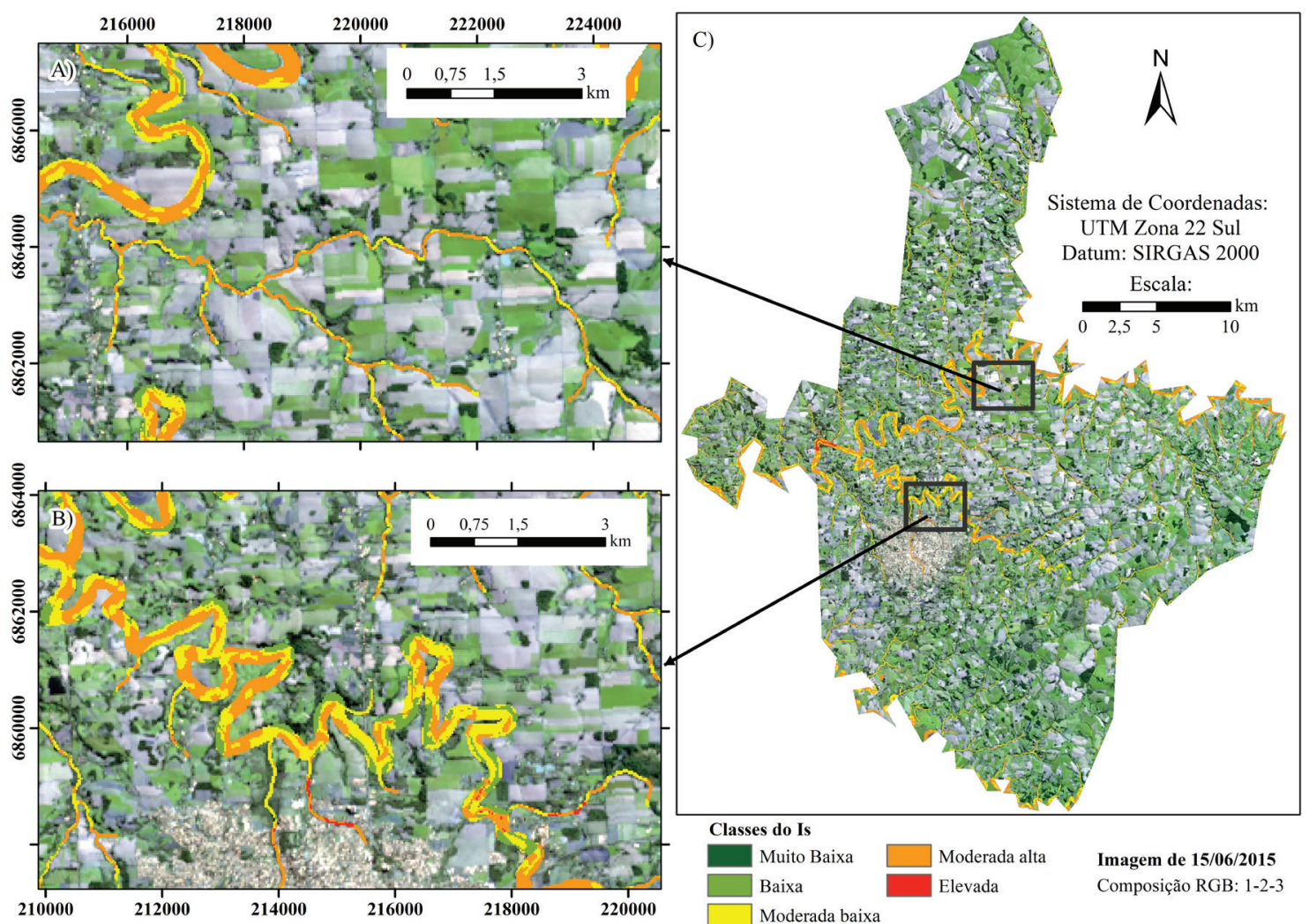

Figura 7. IS dentro das APPh do município de ljuí, RS. A) Ampliação em área Rural; B) Ampliação em área urbana; C) Panorama geral do IS na APPh em ljuí, RS.

Figure 7. IS within the APPh of the municipality of Ijuí, RS. A) Extension in rural area; B) Extension in urban area; C) Overview of IS in APPh in ljui, RS. 
Tabela 8. Áreas referentes às classes de susceptibilidade localizada no interior das APPh do município de ljuí, RS. Table 8. Areas related to the classes of susceptibility located inside the APPh of the municipality of Ijuí, RS.

\begin{tabular}{ccc}
\hline Classes IS & Área (ha) & Área (\%) \\
\hline Muito baixa & 19,571087 & 0,02 \\
Baixa & 1061,455226 & 1,17 \\
Moderada a baixa & 2231,077466 & 2,47 \\
Moderada a alta & 2978,429296 & 3,29 \\
Elevada & 41,74681 & 0,05 \\
\hline
\end{tabular}

Nesse sentido, dentre as principais ferramentas utilizadas para a proteção do meio ambiente no Brasil, a mais recente, e que teve maior destaque é a implantação do Cadastro Ambiental Rural (CAR). Esta ferramenta foi criada com o intuito de integrar as informações ambientais das propriedades e posses rurais, compondo base de dados para controle, monitoramento, planejamento ambiental e econômico e combate ao desmatamento (Brasil, 2012).

Resultados semelhantes foram encontrados por Guimarães \& Carvalho (2013) no município de Moeda em Minas Gerais, onde as APPh do local tinham $50 \%$ de suas áreas não preservadas; os autores ainda citam que esta é a realidade da maioria dos municípios mineiros. Resultados semelhantes foram obtidos por Valle Júnior et al. (2011) no município mineiro de Uberaba, Brasil.

\section{Conclusões}

A partir da aplicação do IS (Ribeiro, 2005), foi possível identificar áreas de maior e menor susceptibilidade à contaminação das águas subterrâneas no município de ljuí-RS, a partir dos cinco parâmetros (recarga, profundidade do nível freático, declividade, material do aquífero e uso do solo), que podem influenciar diretamente na contaminação do aquífero. Tal informação é de grande valor, uma vez que o município de ljuí se encontra em fase de desenvolvimento (Stoffel \& Mattia, 2012), tornando-se uma ferramenta intrínseca na implementação de certos empreendimentos (Foster \& Hirata, 1991).

Mediante a identificação das APPh, e do mapa do IS, foram relacionadas áreas vulneráveis à contaminação. A partir dessa relação, foi comprovada a importância da preservação das APPh. Ressalta-se, portanto, que a melhor maneira de evitar a contaminação dos aquíferos é respeitando as faixas mínimas de preservação ambiental, justificada principalmente por estas estarem localizadas em regiões de maior susceptibilidade à contaminação.

Os resultados apresentados neste estudo também podem servir como uma importante ferramenta para auxiliar órgãos responsáveis pelo meio ambiente, pela gestão de recursos hídricos subterrâneos e licenciamento de atividade potencialmente poluidoras. A realização de estudos desta natureza é importante, pois, além de fornecerem informações ambientais para o suporte da tomada de decisões, agregam à literatura dados que podem servir como parâmetros para o desenvolvimento de novas pesquisas, técnicas, atualização de normas e formação de elementos legislativos que concernem o uso e parcelamento do solo. Sugere-se ainda, que em trabalhos futuros seja investigada a utilização da base de dados do CAR para o cálculo do IS.

Agradecimentos. Os autores agradecem ao CNPq/CAPES pelo auxílio financeiro concedido através de bolsas de Pós-Graduação PPGEAmb e PPGEC/UFSM e bolsas de produtividade.

\section{Referências}

ABAS. Associação Brasileira de Águas Subterrâneas. 2019. Água subterrâneas, o que são? Disponível em: <http://www.abas.org/ aguas-subterraneas-o-que-sao/>. Acesso em: 9 out. 2019.

Aller, L., Bennett, T., Lehr, J. H., Petty, R.J. \& Hackett, G. 1997. Drastic: a standardized system for evaluating groundwater pollution potencial usinghydrogeologic setting. United States: EPA, 202p.

Arcos, F.O., Santos, W.L., Nascimento, F.I.C. \& Galvão, A.S. 2014. Geomorfologia Urbana: o caso do bairro São Francisco - Rio branco - Acre - Brasil. Revista Geonorte, Edição Especial 4, 10(1): 136-140. 
Bergamaschi, H., Dalmago, G.A., Bergonci, J.I., Bianchi, C.A.M., Müller, A.G., Comiran, F. \& Heckler, B.M.M. 2004. Distribuição hídrica no período crítico do milho e produção de grãos. Pesquisa Agropecuária Brasileira, 39(9): 831839.

Borba, W.F., Fernandes, G.D.A., Terra, L.G., Lobler, C.A. \& Silvério da Silva, J.L. 2016. Avaliação da vulnerabilidade intrínseca à contaminação do aquífero na bacia do Rio Passo Fundo no Estado do Rio Grande do Sul. Anuário do Instituto de Geociências, 39(2): 145-154.

Brasil. 2012. Lei $n^{\circ} 12.651$, de 25 de maio de 2012. Dispõe sobre a proteção da vegetação nativa. Disponível em: <http://www2.camara. gov.br/legin/fed/lei/2012/lei-12651-25-maio2012-613076-publicacaooriginal-136199-pl. html.>. Acesso em: 05 mai. 2014

CETESB. Companhia Ambiental do Estado de São Paulo. 2014. Proteção da Qualidade das Águas Subterrâneas. Disponível em: <http://cetesb. sp.gov.br/aguas-subterraneas/informacoesbasicas/protecao-da-qualidade/>. Acesso em 25 de abr. 2016.

Coelho, V.H.R., Montenegro, S.M.G., Almeida, C. das N., Lima, E.R. V. de, Neto, A.R. \& Moura, G.S.S. de. 2013. Dinâmica do uso e ocupação do solo em uma bacia hidrográfica do semiárido brasileiro. R. Bras. Eng. Agríc. Ambiental, 18(1):64-72.

CPRM. Companhia de Pesquisa de Recursos Minerais. 2006. Mapa Geológico do Rio Grande do Sul, Brasília, CPRM/SIAGAS, escala 1:750.000.

Custódio, E. \& Silva Junior, G.C. da. 2008. Conceptos básicos sobre o papel ambiental das águas subterrâneas e os efeitos da sua explotação. Boletín Geológico y Minero, 119: 93-106.

Dambrós, C., Silvério da Silva, J.L. \& Descovi Filho, L.L.V. 2011. Mapeamento da vulnerabilidade do aquífero à contaminação no Município de Rosário do Sul-RS-BR. In: SIMPÓSIO BRASILEIRO DE SENSORIAMENTO REMOTO, 15. 2011, Curitiba.Anais... Curitiba.

p. 3875-3881. Disponível e $\mathrm{m}$ : < http://www.hidro.ufcg.edu.br/twiki/pub/ ASUBO/ASUB-SM/artigo-curitiba.pdf>. Acesso em: 30 set. 2016.
Estado do Rio Grande do Sul. 2002. Decreto $n^{\circ}$ 42.047, de 26 de dezembro de 2002. Regulamenta disposições da Lei no 10.350, de 30 de dezembro de 1994, com alterações, relativas ao gerenciamento e à conservação das águas subterrâneas e dos aquíferos no Estado do Rio Grande do Sul. Diário oficial do Estado, Porto Alegre, RS, 22 dez. 2002. Disponível em:<http://www.charqueadas. rs.gov.br/vigilancia/vig_amb/vigiagua/ Decreto\%2042047.pdf>. Acesso em: 05 de jul. 2016.

Estado do Rio Grande do Sul. 2014. Decreto $n^{\circ}$ 52.035, de 19 de novembro de 2014. Altera o Decreto no 42.047 de 26 de dezembro de 2002, que regulamenta as disposições da Lei $n^{\circ}$ 10.350, de 30 de dezembro de 1994, com alterações, relativas ao gerenciamento e à conservação das águas subterrâneas e dos aquíferos no Estado do Rio Grande do Sul. Diário oficial do Estado, Porto Alegre, RS, 20 nov. 2014. Disponível em:<http://www. al.rs.gov.br/filerepository/repLegis/arquivos/ DEC\%2052.035.pdf>. Acesso em: 08 de dez. 2019.

FEPAM. Fundação Estadual de Proteção Ambiental Henrique Luiz Roessler. 2016. Região Hidrográfica do Uruguai. Disponível em: <http://www.fepam.rs.gov.br/ qualidade/regiao_uruguai.asp >. Acesso: 16 Fev. 2016.

Feron, G.L. \& Reginato, P.A.R. 2014. Avaliação da Vulnerabilidade de Aquíferos Localizados na Região Central de Canoas - RS. Águas Subterrâneas, 28(2): 1-13.

Foster, S. \& Hirata, R. 1991. Determinación del riesgo de contaminación de aguas subterraneas, una metodologia basada en datos existents. Centro Panamericano de Ingenieria Sanitária y Ciências del AmbienteCepis: Lima, 90p.

Foster, S., Hirata, R., Gomes, D., D'elia, M. \& Paris, M. 2002. Groundwater quality protection: a guide for water utilities, municipal authorities, and environment agencies. 1. ed. Washington, DC, World Bank. 114p.

Grabau, Z.J. \& Chen, S. 2016. Influence of longterm corn-soybean crop sequences on soil ecology as indicated by the nematode 
community. Applied Soil Ecology, 100: 172185. Guimarães, F.S. \& Carvalho, G.A. 2013. Determinação de áreas de preservação permanente no município de Moeda - MG, utilizando bases de diferentes escalas topográficas. Caderno de Geografia, 23(39): 22-43.

Hasenack, H. \& Weber, E. (org.). 2010. Base cartográfica vetorial contínua do Rio Grande do Sul - escala 1:50.000. Porto Alegre: UFRGS Centro de Ecologia. Disponível em: < https://www.ufrgs.br/labgeo/index.php/ dados-espaciais/250-base-cartograficavetorial-continua-do-rio-grande-do-sulescala-1-50-000>. Acesso em: 19 Set. 2019.

Hausman, A. 1995. Províncias Hidrogeológicas do Rio Grande do Sul. Acta Geologica Leopoldensia. Série Mapas. São Leopoldo, Brasil: Universidade do Vale do Rio dos Sinos, RS.

IBGE. Instituto Brasileiro de Geografia e Estatística. 2010. Censo demográfico 2010. Brasília. Disponível em: <http://www.cidades. ibge.gov.br/>. Acesso em: 12 Fev. 2016.

Johnston, K., Hoef, J.M.V., Krivoruchko, K. \& Lucas, N. 2003. ArcGIS 9, Using ArcGIS Geostatistical Analyst. ESRI. 300p.

Lindner, M. 2007. Modernização, ações e processos resultantes no setor da produção rural familiar do município de ljuí, RS. Santa Maria, 96p. Monografia de Conclusão de Curso, Curso de Especialização em geociências, Universidade Federal de Santa Maria.

Löbler, C.A. \& Silvério da Silva, J.L. 2015. Vulnerabilidade à contaminação das águas subterrâneas do município de Nova Palma, Rio Grande do Sul, Brasil. Ambiente \& Água-An Interdisciplinary Journal of Applied Science, 10(1): 141-152.

Löbler, C.A., Silvério da Silva, J.L., Martelli, G.V. \& Ertel, T. 2013. Pontos potenciais de contaminação e vulnerabilidade natural das águas subterrâneas do Município de Restinga Seca - RS. Revista Brasileira de Geografia Física, 06(3): 500-509.

Löbler, C.A., Borba, W.F. \& Silvério da Silva, J.L. 2016. Vulnerabilidade das águas subterrâneas da bacia do rio Santa Maria, Rio Grande do Sul. Ciência e Natura, 38: 84-94.
Machado, J.L.F. \& Freitas, M.A. 2005. Mapa hidrogeológico do Estado do Rio Grande do Sul. Porto Alegre, CPRM., escala 1:750.000. (Relatório Final).

Marcuzzo, F.F.N., Andrade, L. \& Melo, D. 2011. Métodos de interpolação matemática no mapeamento de chuvas do Estado do Mato Grosso. Revista Brasileira de Geografia Física, 4(4): 793-04.

Menezes, J.P.C., Bertossi, A.P.A., Santos A.R. dos \& Neves M.A. 2013. Qualidade da água subterrânea para consumo humano e uso agrícola no sul do estado do Espírito Santo. Electronic Journal of Management, Education and Environmental Technology, 17(17): 33183326.

Muhammad A.M., Zhonghua, T., Dawood A.S. \& Earl B. 2015. Evaluation of local groundwater vulnerability based on DRASTIC index method in Lahore, Pakistan. Geofísica Internacional, 54(1): 67-81.

NASA Landsat Program. 2013. Landsat ETM+ cena LC82230802015166LGN00, L1T, USGS, Sioux Falls. Disponível em: <https:// earthexplorer.usgs.gov/>. Acesso em: 10 de mar. 2016.

OEA. Organização dos Estados Americanos., PEA. Programa Estratégico de Ação., GEF. Global Environment Facility. 2009. Síntese Hidrogeológica do Sistema Aquífero Guarani. Série Manuais e documentos Técnicos do Projeto Proteção Ambiental e Desenvolvimento Sustentável do Sistema Aquífero Guarani. Banco Mundial, 102p.

Peluzio, T.M.de O., Dos Santos, A.R. \& Fiedler, N.C. 2010. Mapeamento de áreas de preservação permanente no ArcGIS 9.3. Alegre, Caufes, 62p. Disponível em:<http://www.mundogeomatica. com.br/mapeamentoappsarcgis93.htm >. Acesso em: 23 set. 2016.

PLAMSAB. Plano Municipal de Saneamento Básico do município de ljuí - RS. 2011. Diagnóstico do Saneamento Básico. v.1. Universidade Federal do Rio Grande do Sul, Instituto de Pesquisas Hidráulicas - Porto Alegre: UFRGS. $157 \mathrm{p}$.

Ribeiro, L.F. 2005. Um novo índice de vulnerabilidade específico de aquíferos formulações e aplicações. In: SIMPÓSIO DE 
HIDRÁULICA E RECURSOS HÍDRICOS DOS PAÍSES DE LÍNGUA OFICIAL PORTUGUESA, 7. , 2005, Évora. Anais..., Évora, APRH, p.1-14.

Rossato, M.S. 2011. Os climas do Rio Grande do Sul: sua variabilidade, tendências e tipologia. Porto Alegre, 240p. Tese de Doutorado, Programa de Pós-graduação em Geografia, Universidade Federal do Rio Grande do Sul.

Scheeren, L.W. 2008. Documento DAT-MA No 2849/2008. Rio Grande do Sul: Ministério Público, Divisão de Assessoramento Técnico. 10p. Disponível em: <https://www. mprs.mp.br/media/areas/paibh/arquivos/ diagnostico_bacia_hidrografica_rio_ijui_dat_ complementar.pdf>. Acesso em: 19 set. 2019

SEMA. Secretaria do Meio Ambiente do Estado do Rio Grande do Sul. 2004. Mapa das Bacias Hidrográficas e Municípios do Rio Grande do Sul. Porto Alegre.

Shrestha, S., Semkuyu, D.J. \& Pandey, V.P. 2016. Assessment of groundwater vulnerability and risk to pollution in Kathmandu Valley, Nepal. Science of the Total Environment, 556: 23-35.

SIAGAS. Sistema de Informações de Águas Subterrâneas. CPRM. 2016. Companhia de Pesquisa de Recursos Minerais. Pesquisa geral. Disponível em: <http://siagasweb.cprm. gov.br/layout/>. Acesso em: 2 Fev. 2016.

Simas, L.S.B. 2014. Índices de vulnerabilidade à contaminação de massas de água subterrânea: aplicação à delimitação da Rede Ecológica Nacional no Concelho de Almada (Portugal). Lisboa, 80p. Dissertação de Mestrado, Programa de Pós-Graduação em Geologia Aplicada, Universidade de Lisboa.

Stigter, T.Y., Ribeiro, L. \& Carvalho Dill, A.M.M. 2006. Evaluation of an intrinsic and a specific vulnerability assessment method in comparison with groundwater salinisation and nitrate contamination levels in two agricultural regions in the south of Portugal. Hydrogeology Journal, 14 (1/2): 79-99.
Stoffel, J. \& Mattia, A.A. 2012. O município de ljuí/RS e suas potencialidades econômicas. In: ENCONTRO DE ECONOMIA GAÚCHA, 11., 2012, Porto Alegre. Anais... Porto Alegre, 20p.

Tavares, P.R.L., Castro, M.A.H. de., Costa, C.T.F., Silveira, J.G.P. \& Almeida Junior, F. J. B. 2009. Mapeamento da vulnerabilidade à contaminação das águas subterrâneas localizadas na Bacia Sedimentar do Araripe, Estado do Ceará, Brasil. Revista da Escola de Minas, 62(2): 227-236.

Terra, L.G., Schiavo, B.N.V., Fernandes, G.D.A., Borba, W.F. \& Silvério da Silva, J.L. $2016 a$. Estimativa da vulnerabilidade natural à contaminação do aquífero no município de São Sepé-RS. Revista Monografias Ambientais, 15(1):85-93.

Terra, L.G., Borba, W.F., Fernandes, G.D.A., Trombeta, H.W. \& Silvério da Silva, J.L. 2016b. Caracterização hidroquímica e vulnerabilidade natural à contaminação das águas subterrâneas no município de Ametista do Sul- RS. Revista Monografias Ambientais, 15(1): 94-104.

Valle Junior, R.R., Pedroso Val, B.H., Do Carmo, D.A., Souza, M.A.da C.S. \& Abdala, V.L. 2011. Diagnóstico das áreas de preservação permanente na microbacia hidrográfica do Corrego Jataí. Revista Caatinga, 24(3):153157

Veríssimo, A.C.F. 2010. Hidroquímica, vulnerabilidade e protecção do aquífero de torres vedras. Lisboa, 140p. Dissertação de Mestrado, Programa de Pós-Graduação em Engenharia Geológica, Universidade de Lisboa. 